\title{
Optical clearing mechanisms characterization in muscle
}

\author{
Luís Oliveira* ${ }^{* \dagger}+, \dagger$, M. Inês Carvalho ${ }^{\S}$, Elisabete Nogueira*** \\ and Valery V. Tuchin $\boldsymbol{\Phi}^{, \|, * *}$ \\ *Physics Department - Polytechnic Institute of Porto \\ School of Engineering \\ Rua Dr. António Bernardino de Almeida, 431, 4200-072 Porto, Portugal \\ ${ }^{\dagger} F E U P$ - University of Porto, Rua Dr. Roberto Frias \\ 4200-465 Porto, Portugal \\ ${ }^{*}$ CIETI - Centre of Innovation in Engineering \\ and Industrial Technology, ISEP \\ Rua Dr. António Bernardino de Almeida, 431, 4200-072 Porto, Portugal

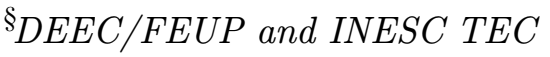 \\ University of Porto, Rua Dr. Roberto Frias \\ 4200-465 Porto, Portugal \\ ${ }^{\top}$ Research-Education Institute of Optics and Biophotonics \\ Saratov National Research State University \\ 83 Astrakhanskaya street, Saratov 410012, Russia \\ "Laboratory of Laser Diagnostics of Technical and Living Systems \\ Institute of Precision Mechanics and Control RAS \\ 24 Rabochaya street, Saratov 410028, Russia \\ **Interdisciplinary Laboratory of Biophotonics \\ Tomsk National Research State University \\ 36 Lenin's avenue, Tomsk 634050, Russia \\ †lmo@isep.ipp.pt
}

Received 31 December 2015

Accepted 7 March 2016

Published 19 April 2016

Optical immersion clearing is a technique that has been widely studied for more than two decades and that is used to originate a temporary transparency effect in biological tissues. If applied in cooperation with clinical methods it provides optimization of diagnosis and treatment procedures. This technique turns biological tissues more transparent through two main

\footnotetext{
$\dagger$ Corresponding author.
}

This is an Open Access article published by World Scientific Publishing Company. It is distributed under the terms of the Creative Commons Attribution 4.0 (CC-BY) License. Further distribution of this work is permitted, provided the original work is properly cited. 
L. Oliveira et al.

mechanisms - tissue dehydration and refractive index (RI) matching between tissue components. Such matching is obtained by partial replacement of interstitial water by a biocompatible agent that presents higher RI and it can be completely reversible by natural rehydration in vivo or by assisted rehydration in ex vivo tissues. Experimental data to characterize and discriminate between the two mechanisms and to find new ones are necessary. Using a simple method, based on collimated transmittance and thickness measurements made from muscle samples under treatment, we have estimated the diffusion properties of glucose, ethylene glycol (EG) and water that were used to perform such characterization and discrimination. Comparing these properties with data from literature that characterize their diffusion in water we have observed that muscle cell membrane permeability limits agent and water diffusion in the muscle. The same experimental data has allowed to calculate the optical clearing (OC) efficiency and make an interpretation of the internal changes that occurred in muscle during the treatments. The same methodology can now be used to perform similar studies with other agents and in other tissues in order to solve engineering problems at design of inexpensive and robust technologies for a considerable improvement of optical tomographic techniques with better contrast and in-depth imaging.

Keywords: Collimated transmittance; agent diffusivity; tissue dehydration; thickness variation; refractive index matching; glucose; ethylene glycol.

\section{Introduction and Basic Concept}

Strong light scattering in most biological tissues limit the application of optical techniques in clinical practice. ${ }^{1}$ The refractive index (RI) mismatch between tissue components originates high scattering coefficients in the visible and NIR spectral ranges. ${ }^{1-6}$ Such high scattering creates power loss in a light beam and limits depth reaching at the area of interest. Considering imaging techniques as an example, low contrast images, obtained from low tissue depths are produced as a consequence of high scattering. ${ }^{7,8}$

The optical immersion clearing technique was proposed to overcome this biological limitation. ${ }^{9}$ This technique is currently applied to minimize the RI mismatch in many biological tissues, since it provides a decrease in the scattering coefficient and an increase in the scattering anisotropy factor, turning the tissues more transparent. It shows great potential to be used in clinical applications. The temporary and reversible tissue transparency allows for reaching deeper tissue layers with reduced beam power loss and minimized beam distortion relative to the natural tissue. Stimulated tissue dehydration (dehydration mechanism) begins as the tissue is immersed in an aqueous solution containing a biocompatible agent. The water that leaves the interstitial fluid (ISF) of the tissue to the outside is replaced by the agent that flows in from the immersing solution (RI matching mechanism). The agent has a higher RI, better matched to the RI of the other tissue components. ${ }^{1,10-15}$ Recent research has demonstrated that this technique is completely reversible and can be used in vivo in cooperation with imaging methods. Some examples are speckle methods to monitor blood flow in dermis or cortical tissues ${ }^{16}$ optical coherence tomography $(\mathrm{OCT})^{17}$ or second harmonic generation (SHG) imaging to improve tissue depth and resolution. ${ }^{18}$

As already indicated above, the two main mechanisms of optical clearing (OC) are designated as tissue dehydration and RI matching. ${ }^{1,6,11,15,19-23}$ Considering in vitro treatments, as the tissue is immersed in the clearing solution, the OC agent (OCA) creates an osmotic pressure over the tissue and stimulates a water displacement towards outside. This dehydration mechanism creates sample shrinkage (lesser sample thickness) and thus more compact and better scatter ordering inside. ${ }^{1,6,15}$ The scattering and absorption coefficients are consequently increased, but due to the smaller sample thickness and constructive interference of the scattered waves in the propagation direction of the incident beam, the sample becomes more transparent. ${ }^{6}$ With the partial loss of water, it turns easier for the OCA molecules to enter the tissue and place themselves in the interstitial locations, close to the scatterers. Since the RI of OCA is higher than the $\mathrm{RI}$ of water, the OCA inclusion in the tissue provides the $\mathrm{RI}$ matching mechanism. ${ }^{15,24}$ An increase 
in tissue transparency is achieved ${ }^{11}$ and better contrast images can be obtained from deeper layers inside the tissue. ${ }^{7}$

Many studies with different kinds of tissues and OCAs like glycerol, ${ }^{25,26}$ glucose $^{11,27}$ or dimethyl sulfoxide ${ }^{14}$ have been made in the last two decades. Some of this research had the objective of clarifying and characterizing the OC mechanisms. The dehydration mechanism in particular, has been widely investigated using different techniques that involves mechanical and temperature variation methods to remove water from tissue area under study. ${ }^{11,28-31}$ These studies reported that the water loss provides a greater tissue transparency through the combination of the smaller sample thickness and the better ordering of scatterers.

Good results were also obtained in studies of the RI matching mechanism. Different clearing potentials have been observed for different concentrations and osmolarities of some particular OCAs tested in skin. ${ }^{19}$ Altered permeability of tissues was also studied to evaluate and optimize the OC mechanism. ${ }^{32}$ These studies have demonstrated that artificially enhanced permeability of tissues leads to different time dependencies for both mechanisms. Quantitative characterization of the OC mechanisms has been tried by measuring optical transmittance and reflectance from porcine skin samples. ${ }^{29}$ Similar studies have demonstrated that different OCA diffusion enhancers (alcohols) provide significant transmittance increase in porcine skin samples. ${ }^{33}$ Sample thickness was also measured in these studies before and after the treatment, showing a small global variation. Other techniques, such as multiphoton microscopy, have also been used to obtain SHG images from ex vivo skin samples under treatment with glycerol solutions. It was observed in these studies the occurrence of collagen dissociation in the extracellular skin matrix. ${ }^{34}$ It was also seen that collagen reassembly can be obtained in post treated samples if rehydrated in saline to wash out glycerol from skin. Other group has also studied the reversibility of protein dissociation to evaluate the OC potential of OCAs such as ethylene glycol (EG), sorbitol or glycerol. ${ }^{35}$ This study has demonstrated that the OC potential of an OCA is directly related to its protein solubility capability. This group found that hyperosmolarity of an OCA relative to tissue forces sample dehydration. In particular, hydrogen bond bridge formation disrupts the collagen hydration layer and facilitates water replacement by the OCA to perform RI matching. ${ }^{36}$ In vivo studies were also performed in dorsal rat skin to evaluate $\mathrm{OC}, \mathrm{OC}$ mechanisms and protein dissociation. ${ }^{37}$ These studies have demonstrated that no collagen fibers were dissociated or fractured at used OCA concentrations. They have showed instead that skin thickness and collagen diameter have decreased as a result of water loss. Another recent study has used a time-lapse multiphoton microscopy technique to elucidate about OC mechanisms. ${ }^{38}$ Using high concentrated glycerol solutions this group has observed that collagen samples have first suffered fast dehydration and shrinkage and that glycerol penetration was slower than dehydration and accompanied with sample swelling.

With the exception of protein dissociation seen in some ex vivo studies, it was always observed that sample dehydration and RI matching mechanisms cooperate as the main driving forces to create the OC effect. Knowing the main OC mechanisms, it is important to individualize them by establishing a quantitative and descriptive characterization for each one. A particular method described in literature $^{6}$ to obtain such individual characterization of OC mechanisms is based on collimated transmittance $\left(T_{c}\right)$ and thickness measurements from ex vivo samples under treatment with OCAs.

Using the theory described in literature,${ }^{6}$ we have performed $T_{c}$ and thickness measurements from muscle samples under treatment with glucose and EG and obtained the diffusion properties of these OCAs and water during OC. ${ }^{15,23}$ Using the time dependence of those measurements and additional data obtained in those studies we develop in this paper a characterization of the OC mechanisms.

In Sec. 2, we present the materials and methods used in our experimental studies, including tissue samples and OCA selection and preparation. Section 3 contains the calculations and discussion of obtained results, the characterization of mechanisms and OC efficiency as a function of time of treatment. Section 4 presents the conclusions and future perspectives for continuing this line of research.

\section{Materials and Methods}

Since the experimental measuring methodology is the same that we have followed in our previous 
studies, ${ }^{15,23}$ we will use this section to make a brief description about the materials used and to describe in detail the calculation procedure that was adopted to obtain the results that characterize the $\mathrm{OC}$ mechanisms and $\mathrm{OC}$ efficiency.

\subsection{Muscle samples}

Skeletal muscle is a fibrous tissue that can be grossly described as a collection of protein fiber chains distributed through the muscle cell cytoplasm (named sarcoplasm) and ISF in-between the muscle cells. ${ }^{39-41}$ This simplified internal description of the muscle indicates that light is strongly scattered inside due to the RI mismatch between sarcoplasm, mitochondria and protein fibers and between IF and muscle cells. ${ }^{42}$ For wavelengths between $600 \mathrm{~nm}$ and $800 \mathrm{~nm}$, literature indicates that the scattering coefficient of skeletal muscle can be dozens of times higher than the absorption coefficient. ${ }^{39}$ Considering this characteristic, we have selected the abdominal wall muscle from the Wistar Han rat to use in our studies. By selecting the abdominal wall muscle, we can retrieve several samples from a single animal sacrifice. In our study, we have used a population of four animals, all adults with ages between 12 weeks and 16 weeks and weights between $450 \mathrm{gm}$ and $500 \mathrm{gm}$.

In each study, after animal sacrifice, the muscle samples were sliced from the abdominal wall muscle block with a cryostat (model HM560 from Thermo Scientific $^{\mathrm{TM}}$, with slicing precision of $50 \mu \mathrm{m}$ for samples with $500 \mu \mathrm{m}$ ). By using all samples from a single animal in a study, maximum physiological similarity between samples was guaranteed. All samples were prepared with circular-slab form with $500 \mu \mathrm{m}$ thickness and a diameter of approximately $10 \mathrm{~mm}$.

To perform thickness measurements during OC treatments with the glucose and EG solutions in different concentrations, we have used bovine muscle samples, also from the abdominal wall area. These samples were prepared with same geometry. Bovine muscle is similar to rat muscle, but has different water contents: $0.709-0.741$ for bovine muscle $^{43}$ and 0.756 for rat muscle. ${ }^{44}$ The results obtained for both muscle samples under treatment with $40 \%$ glucose are very similar, indicating same type of response during $\mathrm{OC}$ treatments.

\subsection{OCAs}

Considering our previous experience, ${ }^{45}$ we have selected glucose and EG to perform our experimental studies. We had previously observed that an aqueous solution of $40 \%$ glucose had good and smooth diffusion in the muscle for a 30 min treatment and that $99 \%$ EG induces a two-stage diffusion and twostage saturation regimes in the same time period. ${ }^{45}$ The fact that $40 \%$ glucose shows a smooth diffusion in a period of $30 \mathrm{~min}$ indicates an optimized diffusion and consequent equilibrium between tissue free-water and solution water content. The twostage diffusion of EG gives the idea that this OCA might produce some significant thickness variations in the muscle. Since we wanted to discriminate the two OC mechanisms and identify possible sample changes, we have selected these two OCAs to use in our studies. Considering the smooth diffusion of $40 \%$ glucose in the muscle, we have selected the following OCA concentrations for glucose and EG: $20 \%, 25 \%, 30 \%, 35 \%, 40 \%, 45 \%, 50 \%, 55 \%$ and $60 \%$. Due to glucose solubility in water, the glucosewater solution with the highest glucose concentration that could be prepared was $54 \%$ glucose. To prepare these various solutions we have used Eqs. (1) in Ref. 15 and (2) in Refs. 6 and 39 to calculate the RI of EG and glucose solutions at $20^{\circ} \mathrm{C}$ and for the wavelength of $589.6 \mathrm{~nm}$ (reference wavelength of the Abbe refractometer):

$$
\begin{gathered}
n_{\mathrm{EG} w}=1.3326+0.001 \times C_{\mathrm{EG}}, \\
n_{g l w}=1.313848+\frac{6.662}{\lambda-129.2}+1.515 \times 10^{-6} \times C_{g l} .
\end{gathered}
$$

In the above two equations, $C_{\mathrm{EG}}$ and $C_{g l}$ represent the EG and glucose concentration in the aqueous solution at $20^{\circ} \mathrm{C}$, respectively. EG solutions were prepared by diluting 99\% EG commercially available in distilled water and controlling solution's RI in the Abbe refractometer. ${ }^{15}$ For glucose solutions, glucose powder was diluted in distilled water and solution's RI was also controlled with the Abbe refractometer.

\subsection{Experimental methodology and calculation procedure}

The experimental and calculated data in this study were obtained both from $T_{c}$ and thickness 


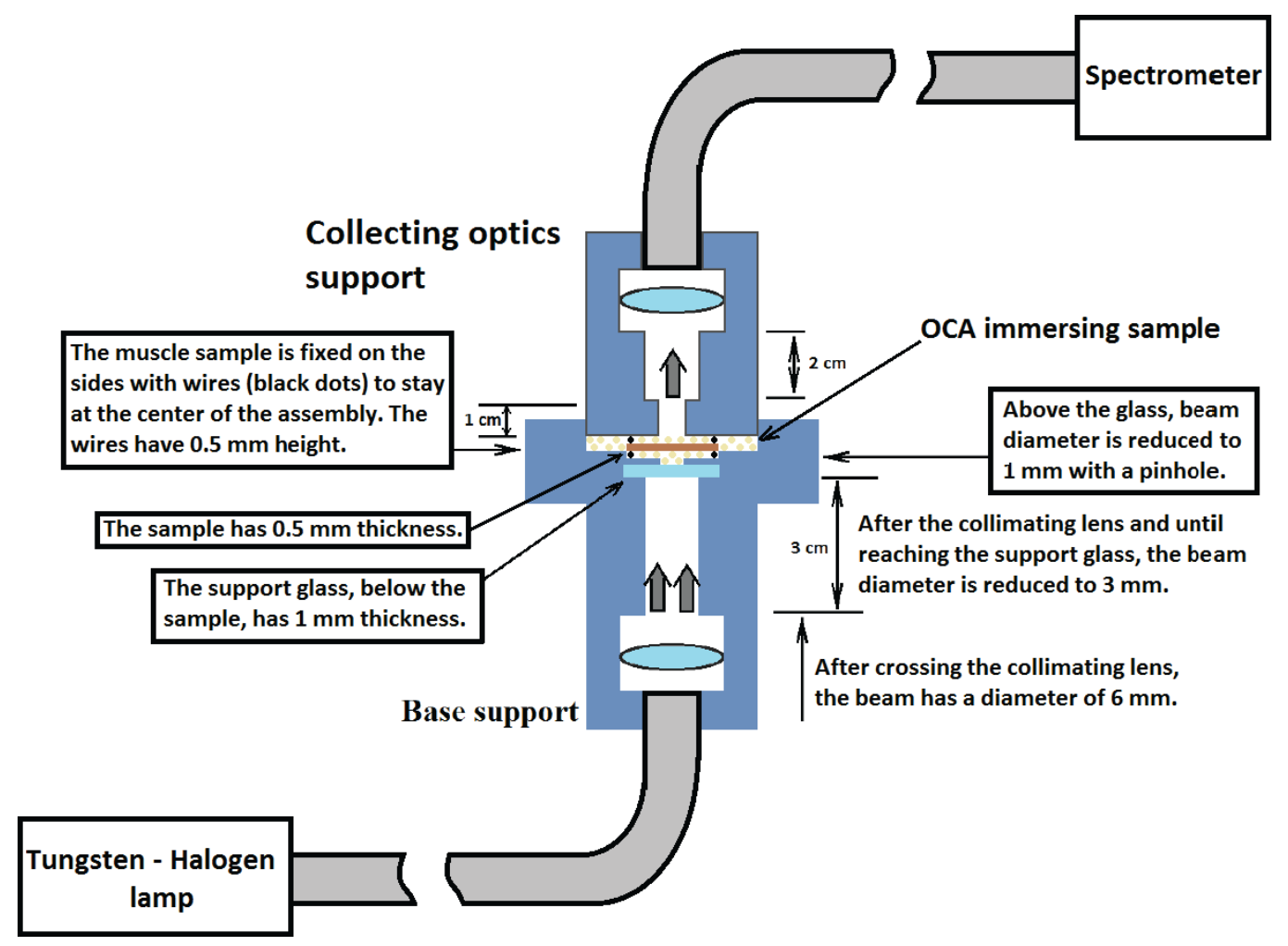

Fig. 1. Collimated transmittance measuring assembly.

experimental measurements made from natural samples and during treatments with different solutions of glucose and EG. Optical $T_{c}$ measurements were obtained with the experimental assembly presented in Fig. 1 and the measuring methodology with this assembly is the same described in our previous publications. ${ }^{15,23}$

Considering the setup presented in Fig. 1, a light beam from a tungsten-halogen lamp is delivered to the lower part of the assembly by an optical fiber cable and collimating lens $(\varnothing=6 \mathrm{~mm})$. An aperture reduces the beam diameter to $3 \mathrm{~mm}$, before crossing the lower supporting glass. Between the lower glass and the sample there is a pinhole to reduce beam diameter to $1 \mathrm{~mm}$. After crossing the sample, the transmitted beam enters the collecting optics support through a central pinhole, also with a diameter of $1 \mathrm{~mm}$. Such transmitted beam is coupled into an optical fiber through a converging lens, to be delivered to the spectrometer. Both optical fibers have a core diameter of $600 \mu \mathrm{m}$. Spectra are produced between $400 \mathrm{~nm}$ and $1000 \mathrm{~nm} \cdot{ }^{15}$

Measurements with the assembly presented in Fig. 1 were made from natural and under treatment muscle samples. During treatments, the sample is kept immersed in the solution, which is maintained in the sample chamber by the lower glass and lateral walls (see Fig. 1). Treatment measurements are initiated as the solution enters the chamber and are maintained at a constant rate during $30 \mathrm{~min}$.

For each particular measurement of natural or under treatment sample, the $T_{c}$ spectra are calculated from the reference $\left(S_{\mathrm{tc}}(\lambda)\right)$ and collimated transmitted spectra acquired from the sample $\left(T_{\mathrm{tc}}(\lambda, t)\right)$ :

$$
T_{c}(\lambda, t)=\frac{T_{\mathrm{tc}}(\lambda, t)}{S_{\mathrm{tc}}(\lambda)} .
$$

Once these calculations were made for the natural sample state and various times of a particular treatment, we have calculated the time dependencies of $T_{c}$ for a collection of wavelengths to evaluate how $T_{c}$ evolves during treatment. Mean $T_{c}$ time dependencies were calculated from the three sets of measurements for the treatments with $40 \%$ glucose and $99 \%$ EG. For the treatments with other concentrations of glucose and EG, $T_{c}$ time-dependency was calculated from a single set of measurements. 
As we have already indicated above for the skeletal muscle, the scattering coefficient is much higher than the absorption coefficient between $600 \mathrm{~nm}$ and $800 \mathrm{~nm}$. For this reason, we have selected the $T_{c}$ time dependencies for individual wavelengths inside this spectral band to proceed with further calculations.

In each treatment, we have identified the beginning of the saturation regime in the selected time dependencies and neglected the remaining $T_{c}$ data from that time until the end of treatment. Such procedure was adopted, since after the beginning of the saturation regime no active flux corresponding to one of the OC mechanisms remains active. For each wavelength within a particular treatment, the time dependency delimited by the beginning of the saturation regime was first vertically displaced to have $T_{\mathrm{c}}=0$ at $t=0$ (natural tissue). The displaced data was then normalized to its highest value, obtaining a set of $T_{c}$ values for each wavelength that varies between zero and unity.

Literature $^{1,6,15,39,46-48}$ indicates that if the volume of solutions used is $10 \times$ higher than sample volume, OCA diffusion into the sample occurs through both slab surfaces during treatment and the averaged OCA concentration $C_{a}(\mathrm{t})$ that is located within the slab sample along a probing light beam (axis $x$ ) at a particular time $t$, is given by:

$$
C_{a}(t)=\frac{1}{d} \int_{0}^{d} C_{a}(x, t) d x \cong C_{a 0}\left[1-\exp \left(-\frac{t}{\tau}\right)\right],
$$

where $C_{a 0}$ is the OCA concentration in the treating solution and $\tau$ is the characteristic diffusion time.

Equation (4) establishes a relation between the time dependence of OCA concentration inside the tissue sample and the characteristic diffusion time of the OCA within the sample. ${ }^{23}$ It can also be used to mimic the OCA concentration inside the sample with the acquired $T_{c}$ measurements.

The various displaced and normalized datasets that correspond to each wavelength were then fitted with a curve that is obtained from Eq. (4), but rearranged as:

$$
T_{c}(\lambda, t)=\frac{C_{a}(t)}{C_{a 0}} \cong\left[1-\exp \left(-\frac{t}{\tau}\right)\right] .
$$

When performing these fittings, we have obtained a $\tau$ value for each wavelength. The mean and standard deviation (SD) of $\tau$ were calculated from the nine values obtained for each treatment. We have used these mean values to represent them graphically as a function of OCA concentration in the immersing solution. The data points in this graph were fitted with a spline to estimate the $\tau$ dependency on OCA concentration, and though identify the values that correspond to the $\mathrm{OC}$ mechanisms.

After estimating the diffusion time of OCAs and water, the correspondent diffusion coefficient can also be calculated for each one. For OCA diffusion into the tissue through both slab surfaces, literature $^{6,9,46,47}$ indicates the relation between the diffusion time $\tau$ and the diffusion coefficient $D_{\text {a }}$ as:

$$
D_{a}=\frac{d^{2}}{\pi^{2} \tau} .
$$

As we can see from Eq. (6), to calculate the diffusion coefficient, sample thickness $d$ is necessary. To proceed with these calculations, we needed the thickness values of the sample at some particular times of treatment. To obtain the necessary sample thickness values to use in the calculation of the diffusion coefficients and also to correlate thickness variation along the treatments with $T_{c}$ data to identify internal changes, we have performed thickness measurements of the samples during the treatments with both OCAs.

Thickness measurements during the treatments were made by inserting the muscle sample inbetween two microscope glasses and measuring the global thickness of this sandwich. Sample thickness was obtained by subtracting the glasses thickness to the global measurements. First, we have measured natural sample's thickness and after that we have injected the immersing solution in-between the microscope glasses to initiate treatment and corresponding measurements. To comply with the $T_{c}$ measurements, we have also used a volume for the solution $10 \times$ greater than the sample volume. Measurements were made very smoothly to avoid undesirable sample compression and to obtain thickness values as true as possible. Three thickness measurement studies were made for each of the treatments with $40 \%$ glucose and 99\% EG and mean values were calculated for a 30 min treatment. For the other treatments, bovine muscle samples were used and a single set of measurements was made in each case. All experimental studies regarding $T_{c}$ and thickness measurements were performed at $20^{\circ} \mathrm{C}$. 
Both the diffusion time and the diffusion coefficient are characteristic to a particular OCA diffusion inside a specific tissue or/and water diffusion outside. Their determination allows characterizing and discriminating between the RI matching/dehydration mechanisms for any tissue/OCA treatment combination.

To perform a more complete characterization of the OC treatments performed, the originally measured $T_{c}$ time dependence data was also used to obtain OC efficiency. Such calculations were made using Eq. (7) for wavelengths between $400 \mathrm{~nm}$ and $1000 \mathrm{~nm}$ :

$$
\mathrm{OC}_{\text {efficiency }}(\lambda, t)=\frac{T_{c}(\lambda, t)-T_{c}(\lambda, t=0)}{T_{c}(\lambda, t=0)} \times 100 \% .
$$

To finalize our calculations, once the diffusion coefficient is known for glucose, EG and water, we can calculate the correspondent viscosity values for these liquids in the muscle. Since the diffusion of OCAs in muscle tissue can be fairly approximated to fluid diffusion in another fluid, we can use the Stokes-Einstein equation to calculate viscosity ${ }^{49}$ :

$$
\eta=\frac{k_{B} T}{6 \pi D r} .
$$

In Eq. (8), $\eta$ represents the liquid's dynamic viscosity in $\mathrm{Kg} /(\mathrm{m} . \mathrm{s}), k_{\mathrm{B}}$ represents Boltzmann's constant with a value of $1.3807 \times 10^{-23} \mathrm{~J} / \mathrm{K} ; T$ is the working temperature in $K, D$ is the diffusion coefficient of the liquid and $r$ is its Stokes radius. ${ }^{49}$

The results from our studies and calculations are presented in Sec. 3.

\section{Results and Discussion}

In this section, we will present our experimental results, calculations and discussion. Since we have obtained various and diverse results from the $T_{c}$ and thickness measurements performed along the OC treatments, we will use the following three subsections to present these results according to each particular subject. In Sec. 3.1, we will present the results obtained for the diffusion and viscosity of glucose, EG and water in the muscle. Section 3.2 will present the results regarding OC efficiency as a function of agent concentration in the immersing solution. Finally, in Sec. 3.3, we will interpret the OC treatments created in muscle by $40 \%$ glucose and $99 \%$ EG, based on the measurements performed and results obtained.

\subsection{Diffusion and viscosity properties of fluids in the muscle}

Using the $T_{c}$ measuring assembly presented in Fig. 1, we have acquired $T_{\mathrm{c}}$ spectra from several natural samples and during the treatments with glucose and EG solutions. Figure 2 presents the mean spectrum of 15 natural muscle samples for visible and near infrared wavelengths:

The increasing behavior of SD with wavelength presented in Fig. 2 is a result of measurements obtained from muscle samples that were collected from different animals and due to different physiology could be more or less transparent at longer wavelengths.

Considering the various treatments performed with glucose and EG solutions, similar measurements were made from the samples along a treatment period of $30 \mathrm{~min}$. For the treatments with $40 \%$ glucose and $99 \% \mathrm{EG}$, three sets of measurements were made, while for the other treatments a single set of measurements was made. For each particular treatment, we have considered the spectra measured in natural state and during treatment and calculated the time dependence of $T_{\mathrm{c}}$ for several wavelengths as a result of the immersion treatment. Figure 3 presents some time dependencies obtained from treatments with glucose solutions:

The wavelengths considered in graphs of Fig. 3 belong to the band between $600 \mathrm{~nm}$ and $800 \mathrm{~nm}$. This band was considered since it is the one

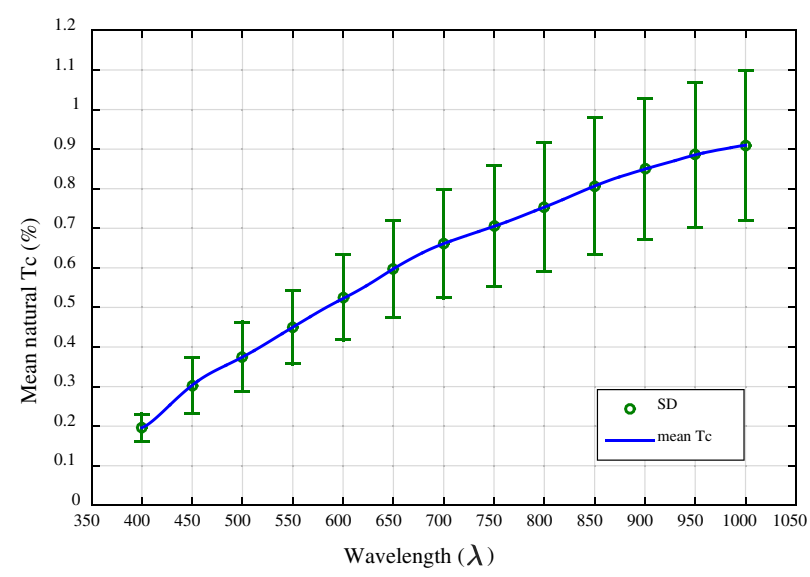

Fig. 2. Collimated transmittance spectrum of rat skeletal muscle (mean of 15 samples; sample thickness $=500 \pm 50 \mu \mathrm{m}$ ). 


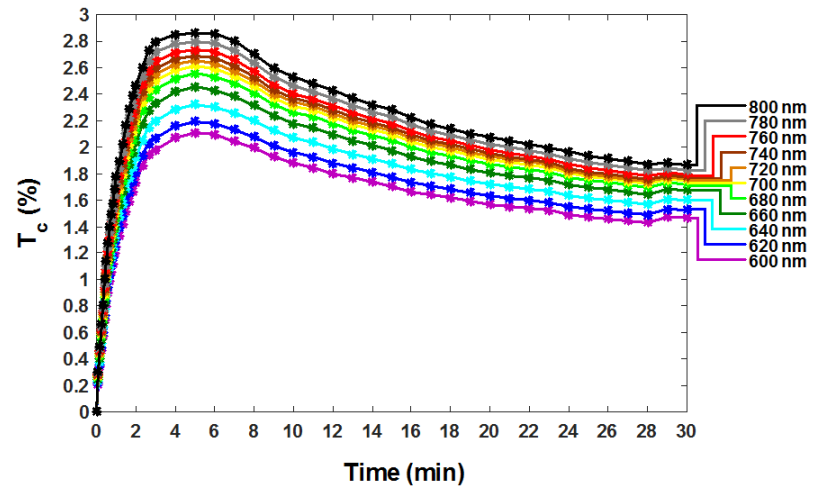

(a)

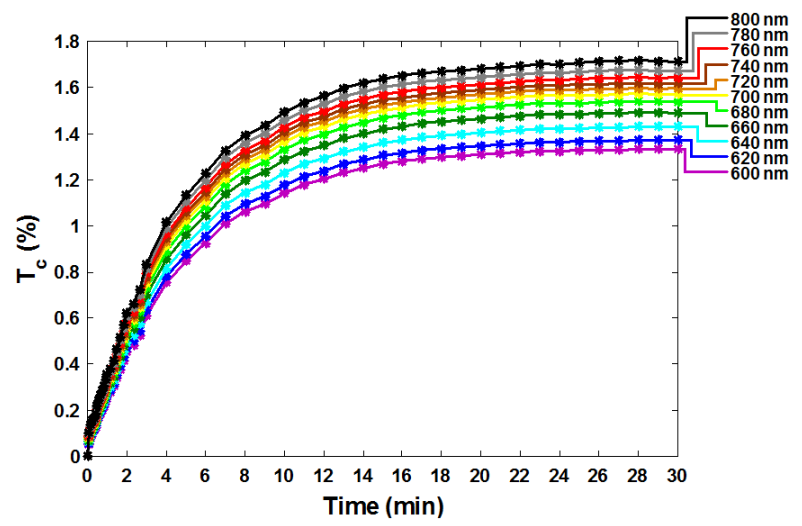

(c)

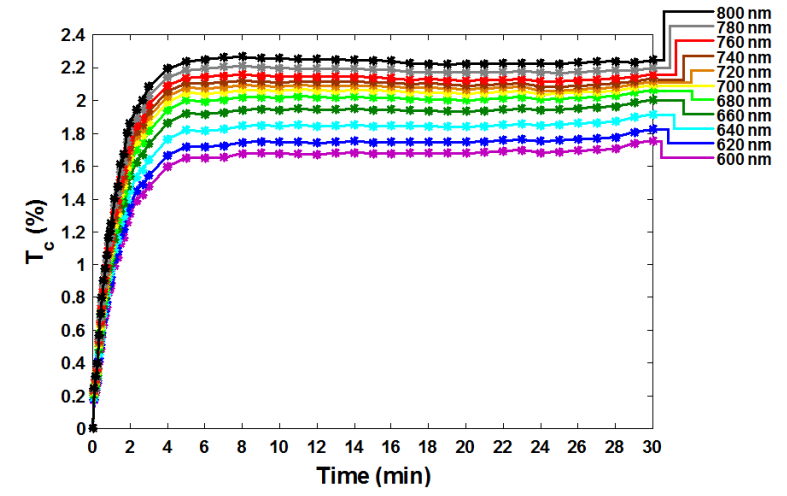

(b)

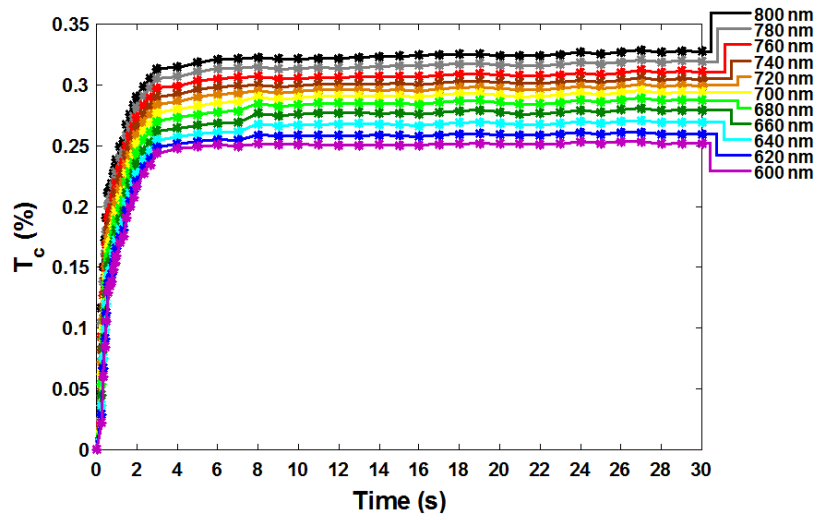

(d)

Fig. 3. Processed raw data from Ref. 23 rescaled to have $T_{\mathrm{c}}=0$ at $t=0$. Treatments with glucose: $20 \%$ (a), 30\% (b), 40\% (c) and $54 \%(\mathrm{~d})$.

where the muscle presents more light scattering in comparison to absorption as we have already mentioned..$^{3,4}$

Figure 3 shows that for treatments with glucose concentrations below $40 \%$, the initial increase in $T_{c}$, before occurrence of saturation is strong and fast. These treatments show a slight decrease in $T_{c}$ after saturation regime is achieved. This fact indicates that both mechanisms occur during the initial stage of OC. We also see from graphs (a) and (b) of Fig. 3 that saturation is reached earlier for the treatment with $20 \%$ glucose, meaning that higher concentrated solutions induce a longer-period of glucose diffusion into the muscle. The magnitude of the decrease in $T_{\mathrm{c}}$ observed during saturation is also stronger in the treatment with $20 \%$ glucose than in the treatment with $30 \%$ glucose. The decrease in $T_{\text {c }}$ observed at later treatment with low concentrated solutions indicates that some water back flux may occur at this later stage of treatment due to the unbalance between solution water and tissue free water.
For the treatment with $40 \%$ glucose, we do not see any decrease in $T_{\mathrm{c}}$. In effect, after approximately $15 \mathrm{~min}$ of treatment, $T_{c}$ is increasing very slowly. This means that for this particular treatment, glucose continues to diffuse into the muscle until the end of treatment.

The treatment with $54 \%$ glucose also shows an initial and fast increase in $T_{c}$ but with significantly smaller magnitude than previous cases, indicating that the dehydration mechanism of OC dominates for such high concentrations. For this treatment, we see a very stable saturation regime, which indicates no effective water or OCA flux at this later stage of treatment.

Similar results for low, intermediate and high OCA concentrations in the immersing solution were obtained in the study with EG. ${ }^{15}$

As part of the diffusion study, by analyzing the graphs of Fig. 3 and others similar for the additional treatments with glucose and EG solutions, we have identified the beginning of the saturation regime for 
each particular case. For each treatment and considering the individual datasets delimited by the beginning of the saturation regime, we have displaced them to have $T_{c}=0$ at $t=0$ (natural tissue), as already represented in Fig. 3. After this vertical displacement, each dataset was normalized to its highest value and fitted with a line described by Eq. (5). From the various fittings made to each dataset of a specific treatment, we have obtained several values for $\tau$ - one for each wavelength. From these values, we have calculated a mean diffusion time for that particular treatment. Such procedure was repeated for all treatments with glucose and EG solutions and the mean diffusion time values obtained are presented in Table 1.

A graphical representation of the data presented in Table 1 was made for each OCA and fitted with a natural spline to evaluate tendency. For both curves, a maximum was observed for an OCA concentration of $40.5 \%$ in the immersing solution. ${ }^{15}$ The confirmation of this OCA concentration by the two studies for different agents is very important, since maximum agent diffusion is imposed by the equilibrium established between free water in the tissue and water in the solution. The free water content in the skeletal muscle of Wistar Han rat was previously unknown, and from these results we could calculate it as $59.5 \%(100 \%-40.5 \%) .{ }^{15} \mathrm{Using}$ this value, we can also calculate the bound water content for the muscle. Since the total water content is reported in literature ${ }^{44}$ as $75.6 \%$, the bound water content is calculated as $16.1 \%$.

The data in Table 1 also gives information about the true diffusion time of glucose and EG molecules and an estimation for the true diffusion time of water in the muscle. For the optimal diffusion

Table 1. Diffusion time values obtained for glucose and EG treatments. ${ }^{15,23}$

\begin{tabular}{lcr}
\hline Glucose/EG & $<\tau_{\text {glw }}>, \mathrm{s}$ & $\left\langle\tau_{\mathrm{EG} w}>, \mathrm{s}\right.$ \\
\hline $20 \%$ & $65.9 \pm 1.8$ & $64.9 \pm 4.6$ \\
$25 \%$ & $72.0 \pm 2.0$ & $76.5 \pm 5.0$ \\
$30 \%$ & $77.3 \pm 2.2$ & $85.3 \pm 5.4$ \\
$35 \%$ & $138.3 \pm 4.3$ & $179.3 \pm 7.9$ \\
$40 \%$ & $300.0 \pm 4.9$ & $442.4 \pm 6.4$ \\
$40.5 \%$ (fitted) & 302.9 & 446.0 \\
$45 \%$ & $209.4 \pm 7.4$ & $283.0 \pm 8.0$ \\
$50 \%$ & $103.6 \pm 7.0$ & $128.7 \pm 6.8$ \\
$54 / 55 \%$ & $58.4 \pm 8.0$ & $81.8 \pm 6.3$ \\
EG 60\% & - & $57.9 \pm 5.2$ \\
\hline
\end{tabular}

concentration (40.5\%), we obtain the maximal value as the true diffusion time of glucose and EG as: $302.9 \mathrm{~s}$ and $446.0 \mathrm{~s}$, respectively. Maximal $\tau$ corresponds to minimal inclusion of fast water flux into measured value. For the treatment with $54 \%$ glucose, we see a diffusion time of $58.4 \mathrm{~s}$, which is representative of the water diffusion time, since at this high glucose concentration, agent diffusion is negligible or even inexistent. Similarly, from the EG data of Table 1, we consider the diffusion time that corresponds to the treatment with $60 \%$ EG as the water diffusion time in the dehydration mechanism. Such value is $57.9 \mathrm{~s}$ and it is a little smaller than the one observed for the treatment with glucose solutions. These values are individualized for each treatment, but they are very similar. Such similarity suggests that independently of the OCA used to create an $\mathrm{OC}$ effect on in vitro muscle samples, the dehydration mechanism is mainly contained within the first minute of treatment.

The diffusion coefficients that characterize the dehydration and RI matching mechanisms can now be calculated from the diffusion time values presented in Table 1. To perform these calculations using Eq. (6) we need to know the sample thickness values at the corresponding times of each treatment, so we will introduce here the thickness measurements that we have performed.

Using the experimental arrangement described in Sec. 2.3, we have made thickness measurements from rat muscle samples under treatment with $40 \%$ glucose and $99 \%$ EG (three sets of measurements for each of these treatments). Similar measurements were made once for each other treatment with bovine muscle samples. Figure 4 presents these results.

The measurements made from bovine muscle samples were performed only once to obtain a comparison between treatments with different concentrations of glucose and EG in solution and establish a relation between the thickness variations observed in rat and bovine muscle.

The first result from Fig. 4 is that bovine and rat muscle show very similar thickness variations when treated with same solution. If we compare data from treatments with $40 \%$ glucose from Fig. 4(a) with data from Fig. 4(c) we see very similar values and time-dependency. On the other hand, comparing between various datasets in Fig. 4(c) we see that as glucose concentration increases in solution, we obtain a higher magnitude decrease in thickness at the beginning of treatment. Such fact indicates that 


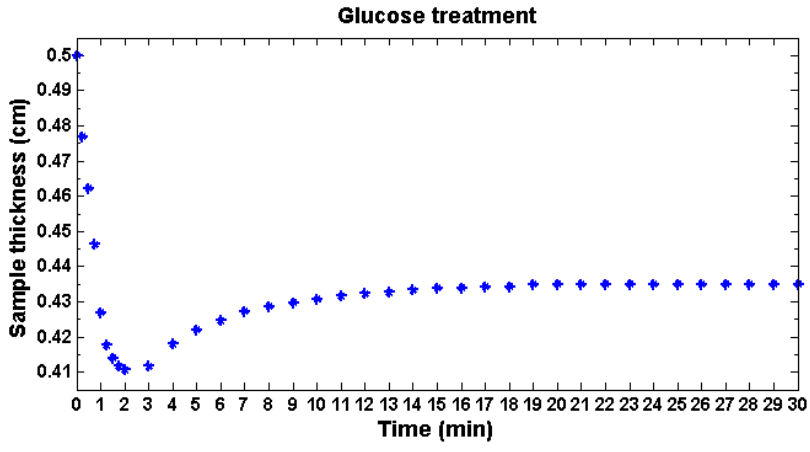

(a)

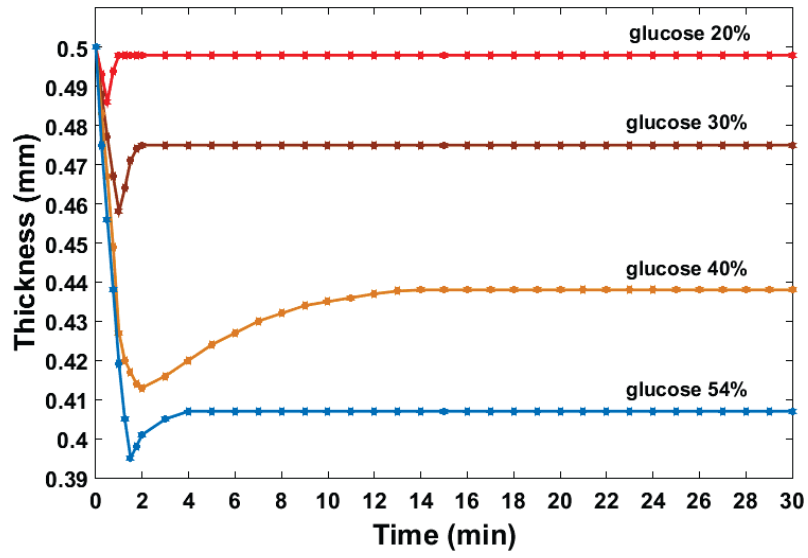

(c)

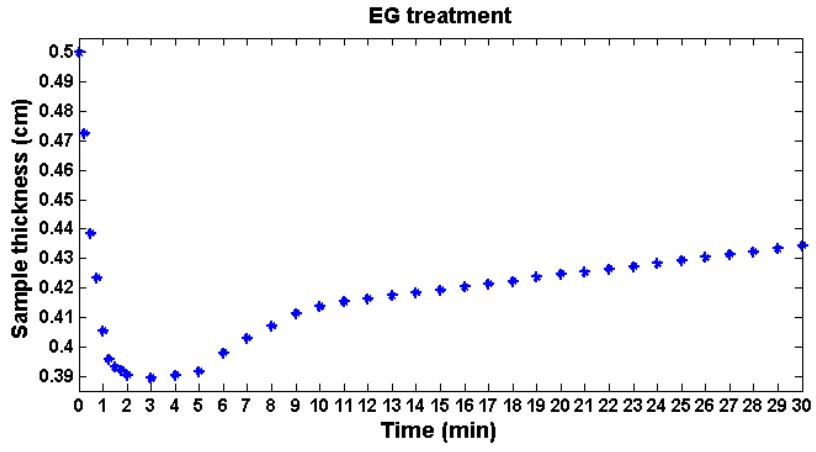

(b)

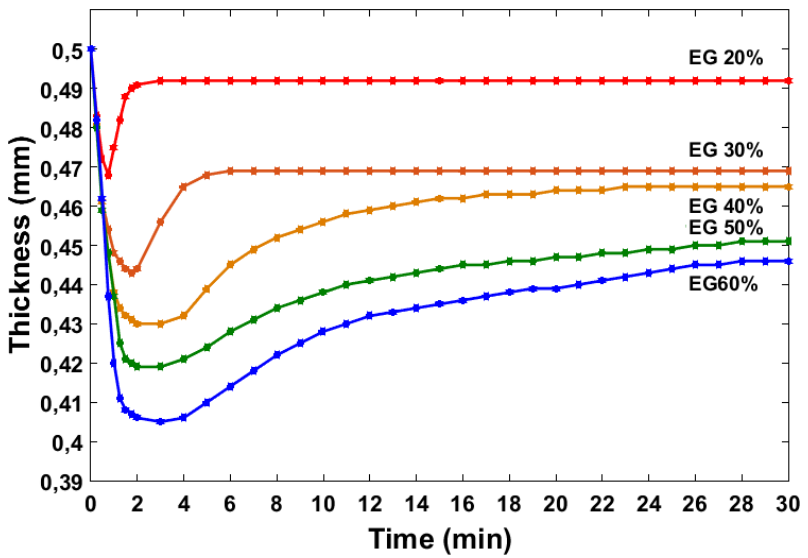

(d)

Fig. 4. Thickness time dependence for: (a) rat muscle treated with $40 \%$ glucose (mean of three studies); (b) rat muscle treated with 99\% EG (mean of three studies); (c) bovine muscle treated with different concentrations of glucose; (d) bovine muscle treated with different concentrations of EG.

higher concentrated immersing solutions provide stronger tissue dehydration.

For the mean thickness variations presented in Figs. 4(a) and 4(b), we have also calculated the SD and observed that this parameter decreases with the time of treatment. For the treatment with $40 \%$ glucose, $3 \mu \mathrm{m}$ SD was observed at $15 \mathrm{~s}$ and $30 \mathrm{~s}$ of treatment, decreasing after that to $1 \mu \mathrm{m}$ and remaining constant until $19 \mathrm{~min}$. After that time, it is practically null. A similar behavior was observed for the SD in the treatment with $99 \%$ EG. At the beginning of that treatment ( $15 \mathrm{~s}$ and $30 \mathrm{~s}$ ), SD was $3 \mu \mathrm{m}$. After that and until $5 \mathrm{~min}$, it decreases to $1 \mu \mathrm{m}$, maintaining this value for the rest of the treatment. The SD values are higher at the beginning of treatments because of tissue compressibility and its variation due to the OC mechanisms. As we know, sample dehydration occurs within the first minutes of treatment as a result of the osmotic pressure created by the OCA in the immersing solution. ${ }^{45}$

The graphs in Figs. 4(a) and 4(b) also indicate the occurrence of sample dehydration at the beginning of treatments. In both cases, we see that sample thickness decreases considerably within the first two minutes. During sample dehydration, if some excessive pressure is made over the sample with the micrometer used in the thickness measuring assembly, it will create extra sample compression that ultimately will lead to an additional amount of water leaving the sample to the outside. Even though we were very careful while performing these measurements, we might have used different compression forces between studies, leading to a high SD during the early treatment stage.

Since thickness is much smaller than superficial area dimensions of the sample, the initial strong thickness decrease seen in graphs of Figs. 4(a) and 
4(b) presents considerable magnitude. In both cases, we see that the initial decrease in sample thickness is contained within the first minute of treatment. This fact correlates well with the water diffusion time values that we have estimated for the treatments with glucose and EG from data in Table $1 .{ }^{15,23}$ The initial strong thickness decrease observed in both treatments indicates that due to water loss during early stage treatment, a more densely packed organization is obtained by muscle fibers (scatterers), which can eventually lead to a temporary slight increase of scattering coefficient (competitive to major reduction of scattering coefficient due to RI matching).

From the graph in Fig. 4(a), we see that the treatment with $40 \%$ glucose induces a turning point in sample thickness at approximately $2.5 \mathrm{~min}$. After that we see some increase in thickness until approximately $8 \mathrm{~min}$, indicating that glucose continues to flow into the interstitial space of the muscle tissue during this time interval. The diffusion time of $302.9 \mathrm{~s}$ that we have obtained for glucose is contained within this $8 \mathrm{~min}$ interval. After $8 \mathrm{~min}$, thickness becomes stable and unchanged, indicating the occurrence of the saturation regime for the rest of the treatment.

For the treatment with $99 \%$ EG, we see from Fig. 4(b) that the turning point in thickness variation occurs at $3 \mathrm{~min}$. Since this solution of EG is almost pure, it is expected that the dehydration mechanism takes more time to be completed than in the case of the treatment with $40 \%$ glucose. Between approximately $3 \mathrm{~min}$ and $8 \mathrm{~min}$ of the $99 \%$ EG treatment, thickness increases, indicating that EG is placing itself in the interstitial locations of the muscle. After $8 \mathrm{~min}$, the observed thickness behavior is different from the one seen for the treatment with $40 \%$ glucose. For the treatment with $99 \%$ EG we see a linear increase in sample thickness, which is evidence that EG continues to flow into the muscle during the remaining of the treatment, but at the expense of some sample swelling.

Using the diffusion time values and corresponding sample thickness for the various treatments in Eq. (2), we can now calculate the diffusion coefficients for glucose, EG and water in the muscle. To calculate the diffusion coefficients of glucose and EG, we need the thickness values measured from muscle samples treated with $40.5 \%$ of OCA concentration in the immersing solution at the times corresponding to the diffusion time values previously obtained. Since we have not performed treatments with solutions containing $40.5 \%$ of OCA and due to the great proximity of this value to $40 \%$, we will use thickness values obtained from treatments with $40 \%$ glucose and $40 \%$ EG. For the case of glucose, we have used in Eq. (6) the sample thickness of $0.042 \mathrm{~cm}$ retrieved from graph in Fig. 4(a) and the diffusion time of $302.9 \mathrm{~s}$ from Table 1 to obtain the diffusion coefficient value of $5.9 \times 10^{-7} \mathrm{~cm}^{2} / \mathrm{s}\left(\right.$ or $\left.5.9 \times 10^{-11} \mathrm{~m}^{2} / \mathrm{s}\right)$.

A similar calculation was made to obtain the diffusion coefficient of EG in muscle. In that calculation, we have used a thickness value of $0.045 \mathrm{~cm}$ that was retrieved from the treatment with $40 \%$ EG presented in the graph in Fig. 4(d) and the diffusion time of $446 \mathrm{~s}$ presented in Table 1 . The resulting diffusion coefficient for $\mathrm{EG}$ in muscle is $4.6 \times 10^{-7} \mathrm{~cm}^{2} / \mathrm{s}\left(\right.$ or $\left.4.6 \times 10^{-11} \mathrm{~m}^{2} / \mathrm{s}\right)$.

Comparing both diffusion coefficients obtained for glucose and EG, we see that tissue matrix limits EG diffusion into the muscle more than it limits glucose. Such difference might also be connected with the difference in viscosity for $40 \%$ glucose and $40 \%$ EG.

Using Eq. (8), we can calculate OCA viscosity within the muscle as:

$$
\begin{aligned}
\eta_{G} & =\frac{k_{B} T}{6 \pi D_{G} r_{G}} \\
& =\frac{\left(1.3807 \times 10^{-23} \mathrm{~J} / \mathrm{K}\right) \times(293 \mathrm{~K})}{6 \pi \times\left(5.9 \times 10^{-11} \mathrm{~m}^{2} / \mathrm{s}\right) \times\left(0.365 \times 10^{-9} \mathrm{~m}\right)} \\
& \cong 1.0 \times 10^{-2} \mathrm{~J} /\left(\mathrm{m}^{3} / \mathrm{s}\right)=1.0 \times 10^{-2} \mathrm{~kg} /(\mathrm{m} . \mathrm{s})
\end{aligned} .
$$

In calculations made with Eqs. (9) and (10), we have used for the Stokes radius of glucose and EG the values of $0.365 \mathrm{~nm}^{50}$ and $0.33 \mathrm{~nm},{ }^{51}$ respectively. The results obtained in these calculations show that EG presents higher viscosity than glucose in muscle. To interpret these results, we can compare the calculated viscosity values for glucose and EG in muscle with the correspondent values of their viscosity in their own media and in water, since water 
is a good comparison model for muscle. It has been reported that pure EG has a viscosity of $1.61 \times$ $10^{-2} \mathrm{~kg} /(\mathrm{m} \cdot \mathrm{s})$ at $25^{\circ} \mathrm{C}$ as it diffuses in its own media. ${ }^{52}$ The viscosity of $40 \%$ EG in water has been reported with the values of $3.1 \times 10^{-3} \mathrm{~kg} /(\mathrm{m} \cdot \mathrm{s})$ at $15^{\circ} \mathrm{C}^{53}$ and $2.15 \times 10^{-3} \mathrm{~kg} /(\mathrm{m} \cdot \mathrm{s})$ at $25^{\circ} \mathrm{C} .{ }^{54} \mathrm{Glu}-$ cose, on the other hand has a dynamic viscosity of $(4.3-6.8) \times 10^{-3} \mathrm{~kg} /(\mathrm{m} \cdot \mathrm{s})$ for temperatures of $25^{\circ} \mathrm{C}-$ $30^{\circ} \mathrm{C} .{ }^{55} 40 \%$ glucose, in particular, has a viscosity in water of $7.2 \times 10^{-3} \mathrm{~kg} /(\mathrm{m} \cdot \mathrm{s})$ at $15^{\circ} \mathrm{C}^{53}$ and a viscosity of $5.19 \times 10^{-3} \mathrm{~kg} /(\mathrm{m} \cdot \mathrm{s})$ at $25^{\circ} \mathrm{C} .{ }^{54}$

Neglecting the temperature differences between our calculated and the reported values, we see that $40 \%$ EG has a dynamic viscosity in muscle a little smaller than the one presented for pure EG diffusing in its own medium and up to seven-fold higher than for EG diffusion in water. $40 \%$ glucose also presents a dynamic viscosity in muscle that is approximately two times higher than values reported for glucose diffusing in water solutions.

The diffusion time, diffusion coefficient and dynamic viscosity values that we have calculated characterize the RI matching and dehydration mechanisms (see Fig. 4) that occur in muscle when treated by glucose and EG solutions, respectively.

To obtain a better characterization of the dehydration mechanism, we can perform similar calculation to obtain the diffusion coefficient of water in the muscle. Since we have already seen that water presents different (although approximated) diffusion time values between the treatments with the two OCAs, we have to make calculations individually for each case. Glucose and EG are two different agents that certainly induce individual and characteristic dehydration mechanisms in muscle tissue.

From the treatment with $54 \%$ glucose we have obtained a diffusion time of $58.4 \mathrm{~s}$ for water. The sample thickness for this treatment and this time (see Fig. $4(\mathrm{c})$ ) is $0.043 \mathrm{~cm}$. Using these values in Eq. (6) we have calculated the diffusion coefficient of water in muscle for glucose treatments as $3.21 \times 10^{-6} \mathrm{~cm}^{2} / \mathrm{s}$.

On the other hand, for the treatment with $60 \%$ EG, we have obtained a diffusion time of $57.9 \mathrm{~s}$. Considering this value and the sample thickness of $0.042 \mathrm{~cm}$ obtained at this time for the same treatment (extrapolated from Fig. 4(d)), we have calculated the diffusion time of water for EG treatments as $3.09 \times 10^{-6} \mathrm{~cm}^{2} / \mathrm{s}$.

The two diffusion coefficients of water obtained for glucose and EG treatments are also very similar.
To get an idea about the magnitude of the diffusion coefficients of water that we have calculated, we can compare them with data from literature. First, we see that both the values are approximately three times smaller than the diffusion coefficient of water in water at $20^{\circ} \mathrm{C}$, i.e., $D_{\mathrm{w} / \mathrm{w}}=8.9 \times 10^{-6} \mathrm{~cm}^{2} /$ s. ${ }^{56}$ The diffusion of water in water is a good model for comparison if we consider that skeletal muscle contains $75.6 \%$ of water. ${ }^{44}$ Since we have obtained a diffusion rate for water three times smaller in muscle than in water, we can assume that muscle cell membrane permeability limits water diffusion.

A similar situation might occur for glucose and EG diffusion in muscle, if we compare our calculated values with data presented in literature. For instance, the diffusion coefficient of EG in water was reported as $1.16 \times 10^{-5} \mathrm{~cm}^{2} / \mathrm{s}$ at $25^{\circ} \mathrm{C} .^{57}$ Ignoring the temperature difference, the diffusion coefficient of EG in muscle that we have calculated is approximately 25 times smaller than the reported value for EG diffusion in water. Such fact suggests that muscle cell membrane permeability slows down EG diffusion significantly inside the muscle. In addition, we might consider that water also diffuses in EG. Literature indicates the diffusion coefficient of water in EG as $1.8 \times 10^{-6} \mathrm{~cm}^{2} / \mathrm{s}$ at $27^{\circ} \mathrm{C} .{ }^{57}$ When performing the OC treatment of muscle with EG, the water that flows out of the tissue will certainly flow through the EG that is flowing in and this water flux out will slow down EG diffusion into the muscle.

To perform a similar analysis for the treatment with glucose, we can consider the reported diffusion coefficient of glucose in water as $5.7 \times 10^{-6} \mathrm{~cm}^{2} / \mathrm{s}$ at $20^{\circ} \mathrm{C} .{ }^{57}$ This value is 10 times higher than the $5.9 \times 10^{-7} \mathrm{~cm}^{2} / \mathrm{s}$ that we have calculated for glucose in muscle at the same temperature. These results show that glucose diffusion (as well as EG diffusion) is limited by muscle cell membrane permeability and possibly by water flowing out through these agents.

Such analysis is very important to understand and characterize the dehydration and the RI matching mechanisms involved in the OC treatments of the skeletal muscle. Such characterization will be presented in Sec. 3.3.

\subsection{OC efficiency of glucose and EG solution in muscle}

After determining the parameters that characterize the OC mechanisms for water, glucose and EG 
treatments, we will now discuss the OC efficiency as a function of glucose concentration in the immersing solution. Considering each of the glucose treatments studied, we have used Eq. (7) to calculate OC efficiency for various wavelengths at different times of treatment. Figure 5 presents these results.

All graphs in Fig. 5 have the OC efficiency for $5 \mathrm{~s}$, $1 \mathrm{~min}, 2 \mathrm{~min}, 5 \mathrm{~min}, 20 \mathrm{~min}$ and $30 \mathrm{~min}$ of treatment.

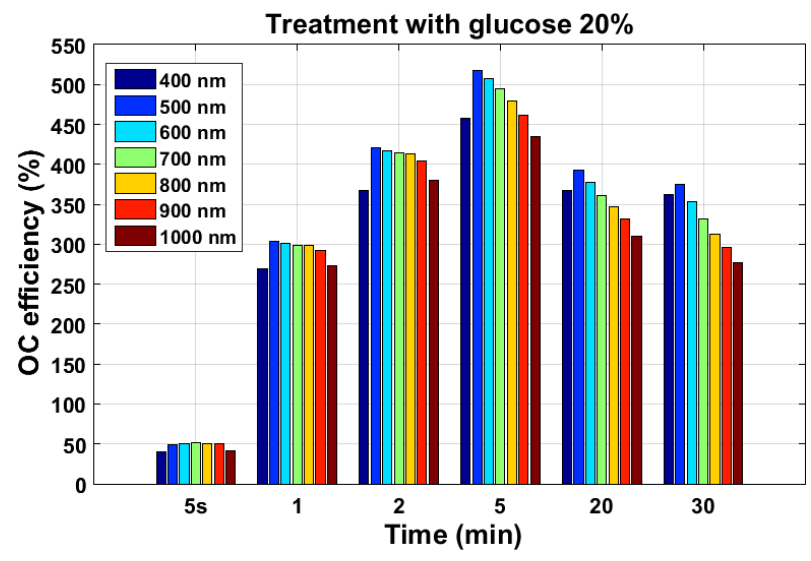

(a)

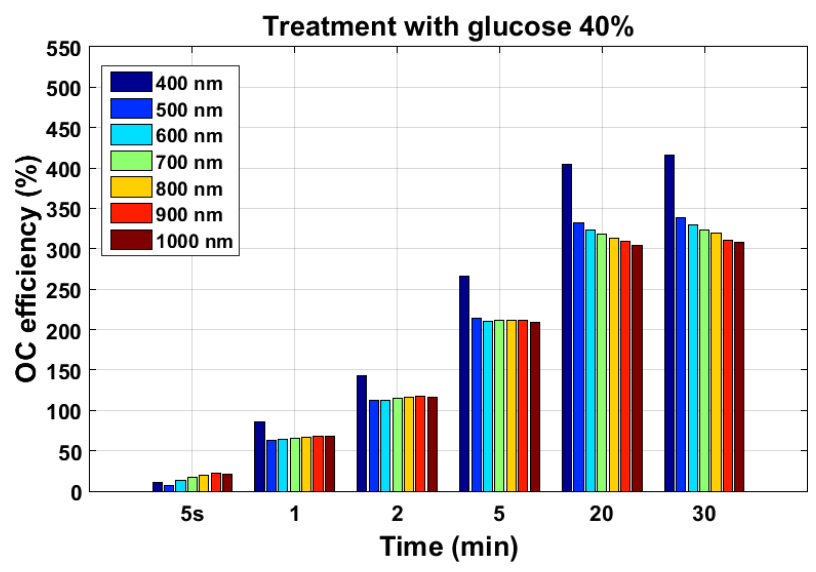

(c)

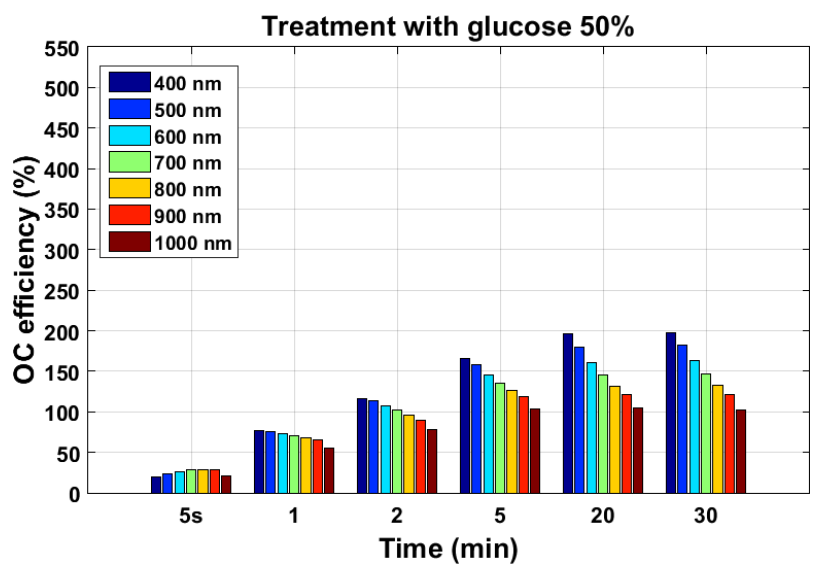

(e)

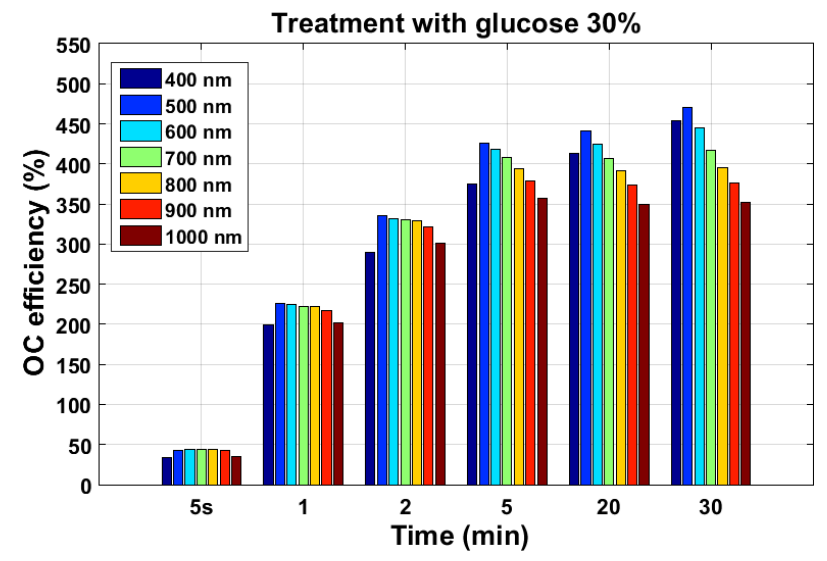

(b)

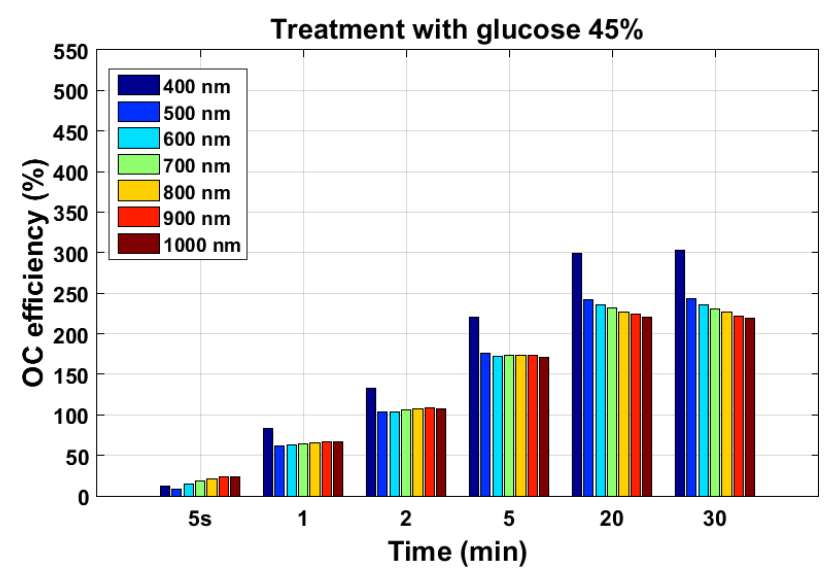

(d)

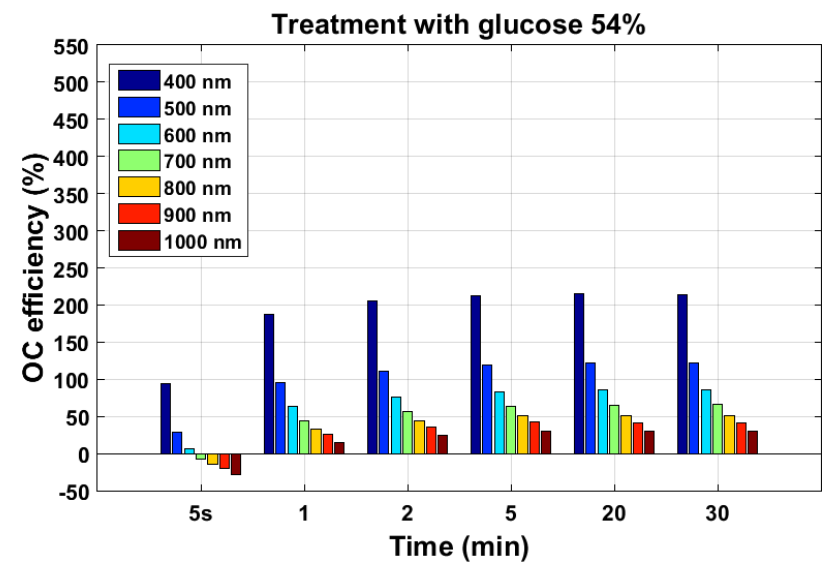

(f)

Fig. 5. OC efficiency for the treatments with glucose: $20 \%$ (a), $30 \%$ (b), $40 \%$ (c), $45 \%$ (d), $50 \%$ (e), and $54 \%$ (f). 
A better analysis of the OC efficiency can be made by presenting results for these particular times.

The various graphs in Fig. 5 show efficiency decrease with wavelength for a particular time of treatment. Such fact is imposed by the spectral form of $T_{c}$ for natural tissue (see Fig. 3) - since lower wavelengths present smaller $T_{c}$ values than longer wavelengths for natural tissue, it is expectable to observe a higher percentage increase for lower wavelengths. This wavelength dependence of the efficiency becomes more evident for longer times of treatment and for highly concentrated solutions. A comparison between all graphs in Fig. 5 shows that the best compromise between increasing efficiency throughout the treatment time and magnitude of the efficiency is seen for the treatment with $20 \%$ glucose. In that case, glucose flows together with water into the tissue during at least the first $5 \mathrm{~min}$ of treatment and provides a fast increase of OC efficiency up to $500 \%$ in the wavelength range 500 $700 \mathrm{~nm}$. However, at latter treatment time OC efficiency decreases due to extra water flux entering the tissue. Nevertheless, it is still high at $30 \mathrm{~min}$ (around 300\%). For 30\% glucose treatment, OC efficiency continuously increases, reaching maximal efficiency of $450 \%$ at $30 \mathrm{~min}$. In the treatment with $40 \%$ glucose, we see a continuous increase during $30 \mathrm{~min}$, up to $300 \%$ for $600-1000 \mathrm{~nm}$ wavelengths. For higher glucose concentrations, the increase in OC efficiency is always maintained during all treatment, but with very much lower levels.

An interesting case is seen for the treatment with $54 \%$ glucose, where for $5 \mathrm{~s}$ of treatment we see negative efficiency for longer wavelengths. Such negative efficiency seems to indicate the occurrence of some tissue darkening at early treatment. Since $54 \%$ glucose is a highly concentrated solution, the osmotic stress created by the glucose in the solution over the sample stimulates fast sample dehydration as we have already seen from thickness measurements presented in Fig. 4. As we have already mentioned, such early significant water loss leads to a more dense packing of tissue scatterers and consequent increase in the absorption and scattering coefficients. In a short time as $5 \mathrm{~s}$, glucose may be already interacting with the outer layers of the tissue, but it does not penetrate much into the sample to initiate the RI matching mechanism. For a longer time of treatment, such as $1 \mathrm{~min}$, glucose has already penetrated in some extent into the tissue and the RI matching mechanism is already occurring, leading to a decrease in the scattering coefficient. Such decrease in scattering is confirmed by the positive efficiency observed at $1 \mathrm{~min}$ of treatment.

Considering still the possible tissue darkening seen at $5 \mathrm{~s}$ for the treatment with $54 \%$ glucose, we observe from Fig. 5(f) that only longer wavelengths (above $600 \mathrm{~nm}$ ) present a negative efficiency. A possible explanation for this difference between shorter and longer wavelengths might be connected with much bigger photon path lengths in the far red and near infrared than in the shorter wavelengths. For this reason, this wavelength range is more sensitive to increase in scattering and absorption coefficient.

Figure 6 presents a similar OC efficiency graph that we have calculated for the treatment with $99 \%$ EG.

In this case, we see no tissue darkening at $5 \mathrm{~s}$ as we did see for the treatment with $54 \%$ glucose, meaning that EG is already performing the RI matching mechanism at this early stage of treatment. Within the first 5 min of treatment OC efficiency presents an almost linear increase. At a late stage of treatment (20 min and $30 \mathrm{~min}), 99 \% \mathrm{EG}$ produces a mean OC efficiency of $650 \%$. Such late treatment increase was not observed for the treatments with glucose and indicates that EG diffuses for a longer time into muscle than glucose, even though with the expense of some tissue swelling as we have observed in Fig. 4(b). Between 20 min and $30 \mathrm{~min}$ we see a small decrease in OC efficiency, possibly due to a second-stage dehydration within the muscle cells. In this treatment, we see that efficiency is a little increasing with wavelength for a particular time of treatment. An exception to this

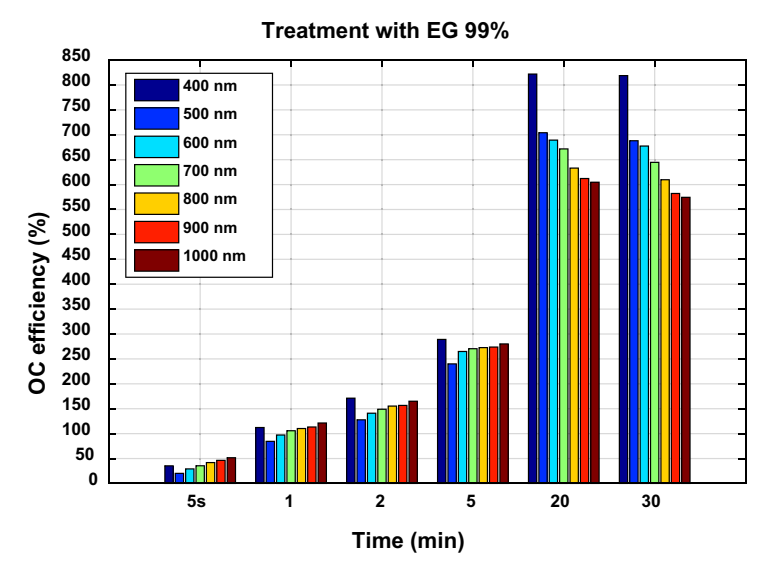

Fig. 6. OC efficiency for the treatment with $99 \%$ EG. 
fact is seen at $20 \mathrm{~min}$ and $30 \mathrm{~min}$, where we finally see a decrease with wavelength as we saw for highly concentrated glucose solutions in Fig. 5. Comparing the levels of OC efficiency between the various glucose treatments presented in Fig. 5 and the treatment with $99 \%$ EG in Fig. 6, we see that EG originates a significantly higher efficiency. Such higher variation might be related not only to the different chemical properties of glucose and EG, but also with the much higher concentration used for the EG solution.

\subsection{Interpretation of muscle $O C$ treatments with $40 \%$ glucose and 99\% $E G$}

We will now consider all previously presented data to analyze and interpret the treatments with $40 \%$ glucose and 99\% EG. To perform this analysis, we will consider the thickness time dependencies presented in Figs. 4(a) and 4(b) and the $T_{c}$ time dependencies presented on Fig. 7:

Both graphs in Fig. 7 present the mean time dependence of $T_{\mathrm{c}}$ for some wavelengths and corresponding SD bars at some times of treatment. When calculating these mean graphs, it was observed in both cases that SD is higher at early time of treatment.

For the treatment with $40 \%$ glucose, we have typical SD values of 0.11 at the beginning of treatment, which decrease to 0.09 at the end of treatment. For the treatment with $99 \%$ EG, we have observed typical SD values of 0.16 at the beginning, which decrease to 0.08 at the end of treatment.

As we can see from graph in Fig. 7(a), $40 \%$ glucose creates a very smooth $T_{\mathrm{c}}$ increase in the muscle due to the water equilibrium between the immersing solution and the free water in the muscle. For this treatment, Fig. 4(a) has showed a fast initial dehydration of the sample as a result of the osmotic stress of the glucose in solution. This does not mean that glucose is not yet diffusing into the muscle at early treatment, but as we have estimated in the diffusion studies, the characteristic diffusion time of glucose in muscle is approximately 5 times greater than the characteristic diffusion time of water. Glucose begins to diffuse as the immersion begins, but at early stage of treatment, glucose molecules interact only with the outer superficial layers of

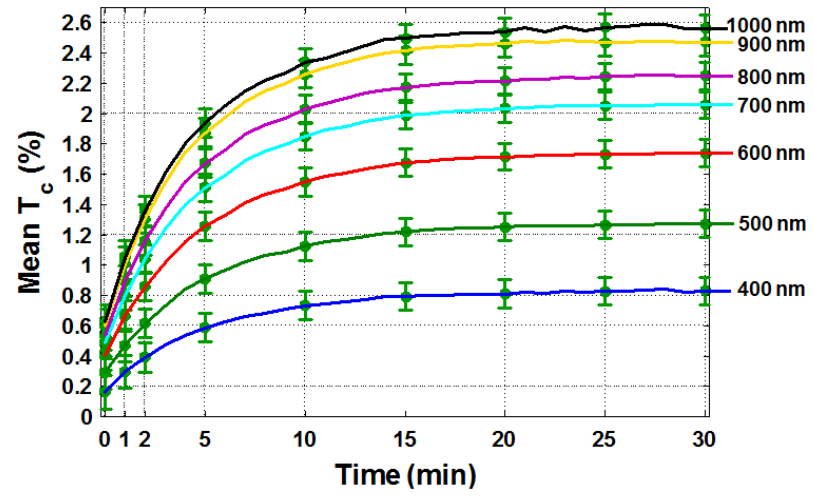

(a)

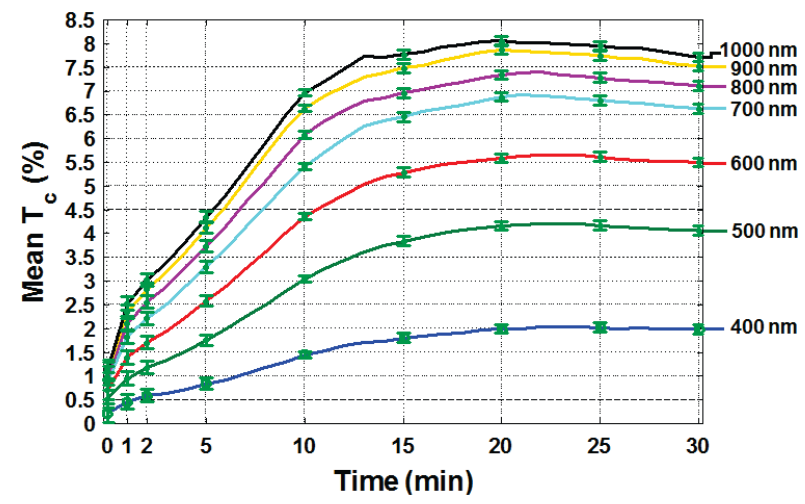

(b)

Fig. 7. Mean time dependence (mean of three studies) of $T_{\mathrm{c}}$ for some wavelengths for the treatments with: (a) $40 \%$ glucose; (b) $99 \%$ EG.

the sample. Consequently, the main OC mechanism at early treatment is sample dehydration as a consequence of the osmotic pressure created by outside glucose over the sample. We have already seen that during tissue dehydration, the loss of part of the free water by the tissue, leads to the approximation of muscle fibers between each other. Such approximation originates a more dense and organized distribution inside the muscle, which creates an increase in the scattering coefficient $\mu_{\mathrm{s}}$ and in the scattering anisotropy factor $g$, providing decrease of the reduced scattering coefficient $\mu_{\mathrm{s}}^{\prime}=\mu_{\mathrm{s}} \cdot(1-g)$.

As water flows out of the sample, glucose diffusion into the muscle becomes easier. Glucose molecules flow through muscle cell membrane into the sarcoplasm and breach into the middle of the group of fibers inside the cell. These fibers, which have previously approached each other as water partially left sarcoplasm, are now forced to separate from 
each other, and the OCA inclusion provides a matching of refractive indices between fibers and surrounding cytoplasm. Such stage of the treatment cannot be identified from the $T_{\mathrm{c}}$ graph in Fig. 7, but it is seen in the thickness graph of Fig. 4(a), between approximately $2 \mathrm{~min}$ and $8 \mathrm{~min}$ of treatment. After $8 \mathrm{~min}$, thickness increase slows significantly and only after $30 \mathrm{~min}$ it is fully stabilized. This indicates that saturation begins and the glucose flux into the muscle stops.

Considering now the $99 \%$ EG treatment, we see that at early stage of treatment it shows similar behavior in thickness and $T_{c}$ to the treatment with $40 \%$ glucose. However, after the first minute of treatment the differences are significant in both measurements. From the graph in Fig. 7(b), we see that $99 \%$ EG creates a two-stage increase in sample's $T_{\mathrm{c}}$. As we have calculated above, EG presents a diffusion time of $446 \mathrm{~s}$, meaning that the EG flux into the tissue is contained within the first $10 \mathrm{~min}$ of treatment and is significantly responsible for the extension of the second-stage rising behavior seen in Fig. 7(b). Nevertheless, this second-stage rising is obtained at the expense of some tissue swelling, as we can see from graph in Fig. 4(b). Figure 7(b) shows that saturation is achieved at approximately $20 \mathrm{~min}$ of treatment and after that $T_{\mathrm{c}}$ slightly decreases. Such $T_{\mathrm{c}}$ decrease obtained at later-stage treatment may indicate the establishment of a second-stage of dehydration within the tissue. Due to a high concentration of EG inside the interstitial locations of the muscle, osmotic pressure created over the muscle cells can induce a consequent conversion of bound water into free water, ${ }^{58}$ leading to dehydration of fibers inside cells. The water that was previously bound is collected in sarcoplasm and gives a slight RI mismatch between more dry fibers and cytoplasm, which leads to scattering increase and some slight drop of transmittance.

Finally, comparing the two graphs in Fig. 7, we see that EG causes a greater increase in $T_{c}$ than glucose. Such increases are approximately of eightfold for the treatment with EG and three-fold for the treatment with glucose. We should bear in mind that the greater increase created by EG is only obtained at the expense of significant changes in the internal structure of the muscle and the choice of highly concentrated solutions should be made after a thorough consideration and analysis.

Based on the results obtained and discussion presented above, we can summarize mechanisms and steps of skeletal muscle tissue $\mathrm{OC}$ as the following:

- The dehydration mechanism provides the loss of water from the muscle cell cytoplasm, thus the muscle fibers inside cells approach each other, leading to a more dense and organized fiber distribution. From one hand, that causes some increase of the scattering and absorption coefficients but, from another hand, gives constructive interference of the scattered waves in the direction of propagation of the incident beam.

- The water loss is also consistent with the thickness decrease that we have observed. Such thickness decrease is significant (up to 20\%) and together with fiber spatial ordering it is responsible for the continuous increase in $T_{c}$, in spite of the slight increase in the scattering coefficient that the dense distribution of fibers originates. We see a continuous increase in $T_{\mathrm{c}}$, since $T_{\mathrm{c}}$ depends on the product of the attenuation coefficient by sample thickness, which decreases as thickness decreases.

- For further increase of OC after 3-5 min of OCA application, RI matching is the leading mechanism, providing a considerable decrease of the scattering coefficient, and correspondingly a further increase of the collimated transmittance.

It was clear in our analysis of the experimental data that some tissue swelling (on the background of the overall tissue sample shrinkage) occurs in such treatments and possibly the conversion of bound to free water that leads to a second and later-stage of tissue dehydration. Such second-stage dehydration occurs within the muscle cells, meaning that muscle fibers become dryer, i.e., a little higher RI mismatch between fibers and sarcoplasm is created, and $T_{\mathrm{c}}$ slightly decreases for longer time of application of highly concentrated OCAs.

\section{Conclusion}

The present study allowed us to perform a quantitative and qualitative characterization of the mechanisms involved in the OC of skeletal muscle. We have used $T_{\mathrm{c}}$ measurements made from samples under treatment with solutions containing different concentrations of glucose and EG to estimate the diffusion time values of these agents in the muscle tissue and characterize the RI matching mechanism of OC. Similar calculations were made to obtain the 
characteristic diffusion time of water in the muscle and characterize the dehydration mechanism. From these values and from the sample's thickness variation data, we can say that both mechanisms of OC begin as the ex vivo sample is immersed in the clearing solution, but each individual mechanism dominates $\mathrm{OC}$ at different stages of the treatment. In both cases studied, we have seen that the dehydration mechanism is confined within the first minute of treatment and it is originated as a consequence of the osmotic pressure created by the agent in the immersion solution. We have also found evidence that the internal structure of muscle changes during the treatments.

From the diffusion studies that we have made, we have discovered that the free water content in the skeletal muscle of rat is $59.5 \%$, a value that was previously unknown. We have also found that for immersing solutions containing this amount of water (like $40 \%$ glucose), OCA diffusion into the muscle is optimized as a consequence of water equilibrium between immersing solution and free water in tissue. In opposition, for immersing solutions containing high OCA concentrations (like $99 \%$ EG), we obtain higher magnitude OC effects, but at the expense of significant changes in the internal structure of the tissue.

The method used in our studies is simple and it has proven adequate to characterize the OC mechanisms of sample dehydration and RI matching. Such method was also productive to obtain additional information about natural (free and bound) water content in soft tissues and about changes that occur in the internal structure due to treatments with particular OCA concentrations. The calculated data of OC efficiency allows the selection of a particular OCA concentration to use in a desired application if we know upfront which increase in $T_{\mathrm{c}}$ we want and how much time we have to obtain such increase.

It is important to note that many of OCAs, such as glucose and EG, are used as solutions for tissue and organ preservation for a short (days) or a long (years) time periods to keep living cells, tissues and organs in condition to be used as future implants. ${ }^{59}$ Therefore, collection of experimental data on diffusivity of molecules, containing in preservation solutions, in various tissues is of great importance for this branch of medical science and technology. ${ }^{60}$ Since we have demonstrated that this simple method provides such results, similar studies can now be performed in other biological tissues or with different OCAs to obtain the necessary diffusion properties for the clinical or low temperature preservation applications.

\section{Acknowledgments}

For the resources made available to perform the experimental measurements, the authors would like to thank to the Physics Department and to CIETI - Center of Innovation in Engineering and Industrial Technology, both located at Polytechnic of Porto - School of Engineering, Porto, Portugal. For the preparation of biological samples, the authors would like to thank to LAIMM - Laboratory of Support to Research in Molecular Medicine from the Department of Experimental Biology, Faculty of Medicine, University of Porto, Porto, Portugal.

VVT is thankful for support from the Government of Russian Federation (Grant No. 14. Z50.31.0004) and The Tomsk State University Academic D.I. Mendeleev Fund Program.

\section{References}

1. V. V. Tuchin, Tissue Optics: Light Scattering Methods and Instruments for Medical Diagnosis, 3rd Edition, SPIE Press, Bellingham (2015).

2. W. Cheong, S. A. Prahl, A. J. Welch, "A review of the optical properties of biological tissues," IEEE J. Quantum Electron. 26(12), 2166-2185 (1990).

3. A. N. Bashkatov, E. A. Genina, V. V. Tuchin, "Optical properties of skin, subcutaneous, and muscle tissues: A review," J. Innov. Opt. Health Sci. 4(1), 9-38 (2011).

4. S. L. Jacques, "Optical properties of biological tissues: A review," Phys. Med. Biol. 58, R37-R61 (2013).

5. J. Mobley, T. Vo-Dinh, Optical properties of tissues, Biomedical Photonics Handbook, T. Vo-Dinh, Ed., pp. 23-75, CRC Press LLC, Boca Raton (2014).

6. V. V. Tuchin, Optical Clearing of Tissues and Blood, SPIE Press, Bellingham (2006).

7. R. Cicchi, F. S. Pavone, D. Massi, D. D. Sampson, "Contrast and depth enhancement in two-photon microscopy of human skin ex vivo by use of optical clearing agents," Opt. Express 13(7), 2337-2344 (2005).

8. X. Wen, S. L. Jacques, V. V. Tuchin, D. Zhu, "Enhanced optical clearing of skin in vivo and OCT in depth imaging," J. Biomed. Opt. 17(6), 066022 (2012). 
9. V. V. Tuchin, I. L. Maksimova, D. A. Zimnyakov, I. L. Kon, A. H. Mavlutov, A. A. Mishin, "Light propagation in tissues with controlled optical properties," J. Biomed. Opt. 2, 401-407 (1997).

10. V. V. Tuchin, "Optical immersion as a new tool for controlling the optical properties of tissues and blood," Laser Phys. 15, 1109-1136 (2005).

11. E. A. Genina, A. N. Bashkatov, V. V. Tuchin, "Tissue optical immersion clearing," Expert Rev. Med. Devices 7(6), 825-842 (2010).

12. K. Becker, N. Jährling, S. Saghafi, R. Weiler, H.-U. Dodt, "Chemical clearing and dehydration of GFP expressing mouse brains," PLoS One 7(3), e33916 (2012).

13. K. Becker, N. Jährling, S. Saghafi, H.-U. Dodt, "Dehydration and clearing of whole mouse brains and dissected hippocampi for ultramicroscopy," Cold Spring Harb. Protoc. 7, 683-684 (2013).

14. D. Zhu, K. V. Larin, Q. Luo, V. V. Tuchin, "Recent progress in tissue optical clearing," Laser Photon. Rev. 7(5), 732-757 (2013).

15. L. Oliveira, M. I. Carvalho, E. Nogueira, V. V. Tuchin, "Diffusion characteristics of ethylene glycol in skeletal muscle," J. Biomed. Opt. 20(5), 051019 (2015).

16. J. Wang, Y. Zhang, P. Li, Q. Luo, D. Zhu, "Review: Tissue optical clearing window for blood flow monitoring," IEEE J. Sel. Top. Quantum Electron. 20(2), 6801112 (2014).

17. K. V. Larin, M. G. Ghosn, A. N. Bashkatov, E. A. Genina, N. A. Trunina, V. V. Tuchin, "Optical clearing for OCT image enhancement and in-depth monitoring of molecular diffusion," IEEE J. Sel. Top. Quantum Electron 18(3), 1244-1259 (2012).

18. O. Nadiarnykh, P. J. Campagnola, SHG and optical clearing, Second Harmonic Generation Imaging, F. Pavone, P. J. Campagnola, Eds., Chap. 8, pp. 169190, CRC Press, Boca Raton (2013).

19. B. Choi, L. Tsu, E. Chen, T. S. Ishak, S. M. Iskhandar, S. Chess, J. S. Nelson, "Determination of chemical agent optical clearing potential using in vitro human skin," Lasers Surg. Med. 36, 72-75 (2005).

20. S. Plotnikov, V. Juneja, A. B. Isaacson, W. A. Mohler, P. J. Campagnola, "Optical clearing for improved contrast in second harmonic generation imaging of skeletal muscle," Biophys. J. 90, 328-339 (2006).

21. X. Wen, V. V. Tuchin, Q. Luo, D. Zhu, "Controlling the scattering of intralipid by using optical clearing agents," Phys. Med. Biol. 54, 6917-6930 (2009).

22. L. Oliveira, A. Lage, M. Pais Clemente, V. V. Tuchin, "Rat muscle opacity decrease due to the osmosis of a simple mixture," J. Biomed. Opt. 15, 055004 (2010).
23. L. Oliveira, M. I. Carvalho, E. Nogueira, V. V. Tuchin, "The characteristic time of glucose diffusion measured for muscle tissue at optical clearing," Laser Phys. 23, 075606 (2013).

24. J. Hirshburg, B. Choi, J. S. Nelson, A. T. Yeh, "Correlation between collagen solubility and skin optical clearing using sugars," Lasers Surg. Med. 39, 140-144 (2007).

25. G. Vargas, E. K. Chan, J. K. Barton, H. G. Rylander, A. J. Welch, "Use of an agent to reduce scattering in skin," Lasers Surg Med. 24, 133-141 (1999).

26. G. Vargas, J. K. Barton, A. J. Welch, "Use of hyperosmotic chemical agent to improve the laser treatment of cutaneous vascular lesions," $J$. Biomed. Opt. 13(2), 021114 (2008).

27. H. Zheng, J. Wang, Q. Ye, Z. Deng, J. Mei, W. Zhou, C. Zhang, J. Tian, "Study on the refractive index matching effect of ultrasound on optical clearing of bio-tissues based on the derivative total reflection method," Biomed. Opt. Express 5(10), 3482-3493 (2014).

28. C. G. Rylander, O. F. Stumpp, T. E. Milner, N. J. Kemp, J. M. Mendenhall, K. R. Diller, A. J. Welch, "Dehydration mechanism of optical clearing in tissue," J. Biomed. Opt. 11(4), 041117 (2006).

29. T. Yu, X. Wen, V. V. Tuchin, Q. Luo, D. Zhu, "Quantitative analysis of dehydration in porcine skin for assessing mechanism of optical clearing," $J$. Biomed. Opt. 16(9), 095002 (2011).

30. A. A. Gurjarpadhye, W. C. Vogt, Y. Liu, C. G. Rylander, "Effect of localized mechanical indentation on skin water content evaluated using OCT," Int. J. Biomed. Imaging 2011, 817250 (2011).

31. Y. Tanaka, A. Kubota, M. Yamato, T. Okano, K. Nishida, "Irreversible optical clearing of sclera by dehydration and cross-linking," Biomaterials 32, 1080-1090 (2011).

32. E. A. Genina, A. N. Bashkatov, A. A. Korobko, E. A. Zubkova, V. V. Tuchin, "Optical clearing of human skin: Comparative study of permeability and dehydration of intact and photothermally perforated skin," J. Biomed. Opt. 13(2), 021102 (2008).

33. Z. Mao, D. Zhu, Y. Hu, X. Wen, Z. Han, "Influence of alcohols on the optical clearing effect of skin in vitro," J. Biomed. Opt. 13(2), 021104 (2008).

34. A. Yeh, B. Choi, J. S. Nelson, B. J. Tromberg, "Reversible dissociation of collagen in tissues," $J$. Invest. Dermatol. 121, 1332-1335 (2003).

35. J. Hirshburg, B. Choi, J. S. Nelson, A. T. Yeh, "Collagen solubility correlates with skin optical clearing," J. Biomed. Opt. 11, 040501 (2006).

36. J. Hirshburg, K. M. Ravikumar, W. Hwang, A. T. Yeh, "Molecular basis for optical clearing of collagenous tissues," J. Biomed. Opt. 15(5), 055002 (2010). 
37. X. Wen, Z. Mao, Z. Han, V. V. Tuchin, D. Zhu, "In vivo skin optical clearing by glycerol solutions: Mechanism," J. Biophotonics 3, 44-52 (2010).

38. V. Hovhannisyan, P.-S. Hu, S.-J. Chen, C.-S. Kim, C.-Y. Dong, "Elucidation of the mechanisms of optical clearing in collagen tissue with multiphoton imaging," J. Biomed. Opt. 18(4), 046004 (2013).

39. A. N. Bashkatov, E. A. Genina, V. V. Tuchin, Measurement of glucose diffusion coefficients in human tissues, Handbook of Optical Sensing of Glucose in Biological Fluids and Tissues, V. V. Tuchin, Ed., Chap. 19, pp. 587-621, CRC Press, London (2009).

40. R. C. Haskell, F. D. Carlson, P. S. Blank, "Form birefringence of muscle," Biophys. J. 56, 401-413 (1989).

41. L. Oliveira, A. Lage, M. Pais Clemente, V. V. Tuchin, "Optical characterization and composition of abdominal wall muscle from rat," Opt. Lasers Eng. 47, 667-672 (2009).

42. V. V. Tuchin, "Tissue optics and photonics: biological tissue structures," J. Biomed. Photon. Eng. 1 (1), 3-21 (2015).

43. A. S. Ginzburg, M. A. Gromov, G. I. Krasovskaya, Handbook on Thermophysical Characteristics of Food Products, Agropromizdat, Moscow (1990).

44. R. F. Reinoso, B. A. Telfer, M. Rowland, "Tissue water content in rats measured by dissecation," $J$. Pharmacol. Toxicol. Methods 38, 87-92 (1997).

45. L. Oliveira, M. I. Carvalho, E. Nogueira, V. V. Tuchin, "Optical measurements of rat muscle samples under treatment with ethylene glycol and glucose," J. Innovative Opt. Health Sci. 6, 1350012 (2013).

46. A. Kotyk, K. Janacek, Membrane Transport: An interdisciplinary Approach, Plenum Press, New York (1977).

47. E. A. Genina, A. N. Bashkatov, V. V. Tuchin, "Glucose-induced optical clearing effects in tissues and blood," Handbook of Optical Sensing of Glucose in Biological Fluids and Tissues, V. V. Tuchin, Ed., Chap. 21, pp. 657-692, CRC Press, London (2009).
48. M. Kreft, M. Lukšič, T. M. Zorec, M. Prebil, R. Zorec, "Diffusion of D-glucose measured in the cytosol of a single astrocyte," Cell. Mol. Life Sci. 70(8), 1483-1492 (2013).

49. H. Suhaimi, S. Wang, T. Thornton, Das D. Bhusan, "On glucose diffusivity of tissue engineering membranes and scaffolds," Chem. Eng. Sci. 126, 244256 (2015).

50. A. Bouchoux, H. Roux-de Balmann, F. Lutin, "Nanofiltration of glucose and sodium lactate solutions: Variations of retention between single- and mixed-solute solutions," J. Membr. Sci. 258, 123$132(2005)$.

51. J. E. Cope, "The Porosity of the cell Wall of Candida albicans," J. Gen. Microbiol. 119, 253-255 (1980).

52. http://en.wikipedia.org/wiki/Ethylene _glycol.

53. http://webserver.dmt.upm.es/ isidoro/bk3/ c07sol/Solution properties.pdf.

54. A. Saluja, D. S. Kalonia, "Measurement of fluid viscosity at microliter volumes using quartz impedance analysis," AAPS Pharm. Sci. Tech. 5(3), 6881 (2004).

55. www.buerkle.de/media/files/Downloads/Viscosity EN.pdf.

56. I. K. Kikoin, Handbook of Physical Values, Atomizdat, Moscow (1976).

57. I. S. Crigor'ev, E. Z. Meilikhova, Handbook of Physical Values, Energoatomizdat, Moscow (1991).

58. B. Schultz, D. Chan, J. Backstrom, M. Rubhausen, "Spectroscopic ellipsometry on biological materials - investigation of hydration dynamics and structural properties," Thin Films 455-456, 731-734 (2004).

59. K. G. Brockbank, M. J. Taylor, Tissue preservation, Advances in Biopreservation, J. G. Baust, J. M. Baust, Eds., Chap. 8, pp. 157-196, CRC Press, Boca Raton (2007).

60. L. M. Alvarez, The official U. S. Defense Department Blog, USA, http://science.dodlive.mil/files/ 2015/01/Organ-and-Tissue-Banking-Compendium2015-Jan.pdf (2015). 